\title{
Chronic C Hepatitis, Interferon and Psychiatric Disorders
}

\author{
Giuseppe Valerio Torres, Emanuela Apicella, \\ Elisa D'Alterio, Angela Chiara Cecere and \\ Antonello Bellomo \\ Additional information is available at the end of the chapter \\ http://dx.doi.org/10.5772/54748
}

\section{Introduction}

The following chapter deals with the clinical management of mental and behavioural disorders that may occur in patients with chronic viral hepatitis $C$ during alpha-interferon therapy.

Actually, in clinical experience, psychopharmacological and psychological interventions are frequently found in patients with HCV-related chronic hepatitis. These interventions are often requested by clinicians with hepatologic and infectious diseases competence, who deliver the ambulatory care for the treatment of interferon in general hospitals.

These interventions are always complex due to several reasons:

a. they are multidisciplinary interventions that require a great collaboration between all specialists involved and high patients management skills.

The clinician has to deal with complex and multi-problematic situations for both the underlying disease and its complications and both the onset of psychiatric disorders, especially anxiety and depressive symptoms. Psychotic symptoms are occasional, instead.

b. there are no specific treatment guidelines for mental disorders related to chronic viral hepatitis. Therefore, the management of these patients can be different according to the internal organization of a psychiatric service and to the one of the general hospital that rarely provide a specific ambulatory care for the treatment and management of psychiatric side effects during alpha-interferon treatment.

c. The clinical picture is often blurred reported and is characterized by heterogeneous symptoms of anxiety and depression that vary from mild to severe degree and may also have a pejorative evolution. 
d. The most common criteria for psychiatric diagnosis used in the international clinical practice and scientific research are those of the DSM IV TR [1]. This means provides diagnostic categories whose inclusion criteria are very restrictive. Mental disorders due to a general medical condition and substance-induced mental disorders are included in these diagnostic categories. This last diagnostic category even includes mental disorders induced by drugs. Nevertheless, there is no explicit reference to either HCV or interferon therapy in both diagnostic categories.

Moreover, the underlying causes of mental disorders in patients with chronic HCV hepatitis seem to be very complex.

In Psychiatry it is widely recognized as valid the bio-psycho-social paradigm according to which the causes of mental illness must be found not only in genetic and constitutional reasons but also in psychological and environmental factors. This paradigm is even more appropriate when the mental illness and the organic illness are present at the same time.

The psychological impact of diagnosis of HCV-related chronic hepatitis, interferon treatment and hcv neurotoxicity are definitely important in the genesis of symptoms.

\section{HCV infection as life event}

The role of stressful psychosocial events or "life events" in the unleashing of psychic pathology is generally accepted by most clinicians. As a matter of fact, it is difficult to document a causal link between life events and mental illness and it is hard to think of a real direct causality between these two factors. Some authors have proposed a model based on a circular relationship between the event and the psychopathological manifestation, rather than a model based on a linear cause-effect relationship [2]. Available researches, moreover, show that only some individuals will develop a specific psychiatric disorder at the presence of the same life events [3].

Different regulation scales are used to define the events that can be considered as stressful and to value their psychological burden. A personal illness, especially if chronic, is certainly an event characterized by great emotional and psychological impact [4]. Moreover, symptoms such as mood changes and anxiety, which are commonly observed in patients affected by HCV, can be related to the awareness of suffering from a chronic progressive disease and to the past or current substance abuse [5].

As we have said, the aetiology of psychiatric disorders is multifactorial and several factors can lead to the development of the manifestations: genetic factors, evolutionary factors, environmental factors and life events. The onset of an organic diseases can lead to the outbreak of a psychiatric disorder, not only for the psychological impact that the disease has but even due to the changes in the immune system that can establish in certain medical conditions.

Depressive symptoms are often found in pathologies such as AIDS [6], diabetes mellitus [7], multiple sclerosis [8] and cardiovascular disorders [9]. 


\section{Proinflammatory cytokines and neurotransmitter systems}

It is well known that the assumption of interferon $\alpha$ (IFN $\alpha$ ) is associated with the onset of depressive symptoms, similar to those observed in major depression [10], in fact, from $30 \%$ to $50 \%$ of patients receiving IFN develop depression during treatment [11].Many data in literature show the very important role of pro-inflammatory cytokines in the onset of the Depression [12,13-14,15].Since the 90s, several studies have highlighted that in certain cases the alterations of the immune system can contribute to the development of depressive symptoms, favouring the acquisition of new knowledge about the so-called Psychoneuroimmunology [16].

The Neuro- Endocrine System (SNE) and the Immune System (SI) are part of a totally integrated biological circuit, in fact, the same biochemical signals are used both for the exchange of information between the components of the same system both for communications between the two systems. Numerous researches, in fact, have shown that the SI is able to modify its responses, either through automatic mechanisms of regulation either by signals coming from the Central Nervous System (CNS) and endocrine system (SE). In turn, the cells of the SI, sending signals to the central nervous system and to the SE, trigger specific neuroendocrine and behavioural responses. Pro-inflammatory cytokines are ones of the main messengers employed by SI to communicate directly with the SNE, in particular, they stimulate the activity of the locus coeruleus (LC) and the hypothalamic secretion of Corticotrophin-Releasing Factor (CRF) [17].

The immune cells communicate with the SNE also by means of several neuropeptides and neuro-hormones, such as the CRF, adrenocorticotropic hormone (ACTH), the Growth Hormone (GH), the Thyrotropin-Releasing Hormone (TRH), prolactin, the $\beta$-endorphin, etc.; immune cells, in fact, possess specific membrane receptors, both for these mediators both for neurotransmitters such as adrenaline, serotonin, histamine, GABA [18].

The existence of a bi-directional network of communication between SNE and SI is extremely important since it allows to SNE to receive and respond to a wide range of non-cognitive stimuli, which include stresses-induced stimuli and infectious, autoimmune or neoplastic nature-stimuli, that it could not perceive otherwise. The relationship between the nervous system, endocrine system and immune system has been highlighted by several studies. The baseline concentration of soluble receptors for TNF and IL- 6 were linked with the development of depression during therapy with IFN $\alpha$ [19]. Moreover it was found that patients suffering from cancer and depression showed higher levels of interleukin 6 (IL6) compared to patients affected by cancer but without depression [20]. In patients suffering from $\mathrm{HCV}$, concentrations of receptors for IL-2, IL-6 and IL-10 are significantly higher in patients who develop a depressive symptomatology during therapy with IFN, compared to patients who did not develop it. Therefore, a greater activation of the immune system may be predictive of the development of depression during IFN therapy [21,22].

Let's look now at the way through pro-inflammatory cytokines, in particular IFN $\alpha$, act in the genesis of psychiatric interest symptoms. It's well known that serotonergic and 
dopaminergic neurotransmitter systems are involved in depressive disorders and anxiety disorders. In clinical practice, drugs that act on the neurotransmitter systems are commonly used. The role of IFN $\alpha$ in the development of depression and anxiety may be partly due to the action on serotonin metabolism. The administration of selective serotonin reuptake inhibitors (SSRIs), in fact, inhibits the development of depression in patients treated with IFN $\alpha$ [22]. The administration of paroxetine (SSRI) reduces to approximately 4 times the risk of onset of depression during therapy with IFN $\alpha$ [23]. The IFN $\alpha$ and cytokines in general, would contribute to the alteration of serotonin metabolism through the activation of an enzyme called indoleamine 2-3 deoxigenase (IDO), which converts tryptophan, an amino acid precursor of serotonin, in kynurenine [24], leading to a reduction in the synthesis of serotonin. In fact, it has been demonstrated that, patients treated with IFN $\alpha$, who develop depression, have lower blood concentrations of tryptophan $[25,26]$. Patients who develop major depression during IFN- $\alpha$ therapy, compared with patients who did not develop, show in the peripheral blood significantly reduced level of tryptophan and increased levels of kynurenine [27]. These data would support the hypothesis according to which the reduction of levels of tryptophan and, consequently the reduction of the synthesis of serotonin, are consequent to activation of the IDO.

Another mechanism involved in the reduction of levels of serotonin induced by cytokines is the activation of a protein kinase, called MAPK, by themselves.

The IFN $\alpha$ is a potent inducer of p38 MAPK; the activation of this protein kinase increases the expression and synthesis of serotonin transporters [28,29], thus resulting in the increase of serotonin reuptake, with consequent reduction of the neurotransmitter in the synaptic cleft. Recent studies have linked the increased function of serotonin transporters with seasonal affective disorder [30].In addition to the reduction of serotonin, alterations in metabolism of dopamine (DA), would also contribute to the onset of symptoms such as psychomotor retardation and fatigue. In fact, the IFN and, more generally, pro-inflammatory cytokines, act on the metabolism of DA, especially at the level of the basal ganglia, causing a symptomatology characterized by neuro-vegetative symptoms, anhedonia, psychomotor retardation, fatigue [31,32-33].

Dopamine is involved in different circuits in mood, reward and motivation, motor activity, sleep-wake rhythm, cognitive processes [34,35].

It has been shown that IFN $\alpha$ induces motor slowing and this slowing down is correlated, significantly, to the development of depression and fatigue [31].

The mechanisms by which the IFN would act resulting in a reduction of DA in the synaptic cleft are manifold; primarily cytokines, and therefore the IFN $\alpha$ as well, induce an increase of nitric oxide (NO), which causes a reduction of tetrahydrobiopterin (BH4) that is a co-enzyme for tyrosine hydroxylase, which converts tyrosine to L-DOPA, this causes a reduction in the synthesis of DA [27].

It has also been shown that the action of IFN on BH4 is mediated by nitric oxide, in fact, treatments that inhibit the synthesis of NO, block the inhibitory effect mediated by IFN on the 
concentration, at the level of the central nervous system, of tetrahydrobiopterin ( BH4) and DA [36]. It was also demonstrated that the IL6 (whose blood concentrations increase after administration of IFN $\alpha$ ), reduces the tetrahydrobiopterin at the neuronal level [37].

Another mechanism by which the IFN $\alpha$ works reducing the levels of DA in the synaptic cleft involves the Kynurenic acid (KA). As we have already said, with regard to the effect of cytokines on the metabolism of serotonin, the IFN determines an activation of the enzyme indoleamine 2,3 dioxygenase (IDO), which cleaves the tryptophan in kynurenic acid (KA). KA reduces the release of glutamate. The glutamate, as well known, stimulates the release of dopamine [37,38-39]. The final effect is, in this case as well, a reduction of the levels of DA in the synaptic cleft. Finally, the IFN $\alpha$, as we mentioned above, can activate some protein kinase, the MAPK pathway, which results in the increase in the expression of those genes coding for transporters of DA, this causes an increased DA reuptake with consequent reduction of the available levels of DA in the synaptic cleft [40].

It is worth pointing out that, in addition to depressive and autonomic manifestations, symptoms of anxiety and irritability may also occur during therapy with IFN $\alpha$.

Several studies have shown that a significant number of patients develop anxiety symptoms 1 or 2 hours after taking the interferon therapy [41]. Some neuroimaging studies have shown that one of the mechanisms by which cytokines may cause anxiety and hyper arousal, is related to the increased activation of neural circuits in dorsal anterior cingulate cortex $[42,43]$.

\section{Neurotoxicity of $\mathrm{HCV}$}

With regard to the aetio- pathogenesis the third aspect to consider is the one of specific neurotoxicity of HCV.

In the last years it has been supposed that psychiatric disorders that occur in patients affected by $\mathrm{HCV}$ are due to a direct effect of the virus.

In particular, it has been suggested that there is no link between the onset of depressive disorders in patients with HCV infection and the IFN $\alpha$ - therapy [44].

An interesting study using magnetic resonance spectroscopy (MRS), a non-invasive technique that allows to get localized biochemical information from tissues and organs, has pointed out several alterations of cerebral metabolites in patients with $\mathrm{HCV}$ infection [45]. These alterations are very similar to those caused by HIV virus which can be found in the central nervous system during the overt disease. $[46,47]$. These studies support the hypothesis according to which, even the HCV would be able to infect cells of the central nervous system, causing changes in the brain [48]. According to this hypothesis, the virus after infecting monocytes in circulation, is introduced into the CNS thanks to a mechanism known as "Trojan Horse," resulting in neuronal dysfunction. This hypothesis is supported by recent studies that have demonstrated the presence of HCV gene sequences in post-mortem examined brain tissue [49]. Another important aspect that rises from data in literature is that different viral genotypes correlate 
with a different response to the antiviral therapy with IFN- $\alpha$. Up to now, according to differences in nucleotide sequence, 7 different genotypes of HCV (in turn subdivided into several subtypes) have been identified, with a different geographical prevalence. The most common genotypes in Europe are 1a, 1b, 2a, 2b and 3 while in the USA is genotype 1a. Many people believe that the genetic heterogeneity of the virus strains is the basis of the differences in the development of disease and in the responsiveness to treatment. It is well known, in fact, that the genotypes 1, 5 and 6 have a lower response to antiviral treatment. In particular, the virus $1 \mathrm{~b}$ leads to a more rapid progression to cirrhosis. In contrast, viral genotypes 2 and 3 have a better response to antiviral treatment [50].

\section{Psychological aspects of the diagnosis of hepatitis $C$ and consultation - Liaison psychiatry}

What has been argued so far gives an account of how much weight may have, in a bio-psychosocial dimension, the diagnosis of HCV. The patient has, in fact, to live a chronic medical condition, often without any physical and psychological resource to manage it, whose treatment is not always effective and has serious side effects; in addition, an uncontrollable factor, the constitutive genetic component indeed, may be decisive to a successful treatment.

The meeting with an illness, whatever it is, brings about such a big change of life to threaten the own sense of identity so that the disease can be lived with deep anguish, hate, anxiety and depression.

Organic disease, whatever is the form in which it may manifest, acute or chronic, mild or severe, always represents a more or less significant break of the individual cycle of life and therefore, it involves the psychological sphere.

Several times we have emphasized the complexity of the Psychiatrist role in patients with HCV-related chronic hepatitis. Actually, these patients live a condition of severe insecurity, both physical and psychological. They are forced to change their lifestyle, putting in practice new behaviours and careful conduct rules in personal, social and relational context.

They live together with the fear of serious complications and death. The many disorders related to hepatitis, depressive symptoms and interferon treatment, make them lose their physical and mental energy to manage their disease condition. Typically, in fact, depressive symptoms reported by this type of patients, are purely somatic; fatigue, muscle ache, tiredness are the most common symptoms that cause great discomfort to patients and most influence their quality of life. They are "carriers" of a deep sufferance but they have no more own energy to deal with it. It is clear that these people are able to encourage the clinician to work harder in order to match medical experience with psychological and relational competence. They are also able to lead the clinician to overcome the socalled "mind-body dichotomy" and get a new clinic view focused on the suffering person and not just on the disease. This aspect is really important because it makes actual an ethical problem that all health care professionals have to face in everyday clinical life: 
a sterile biological reductionism to the detriment of the centrality of the person [51]. The Consultation and Liaison Psychiatry deals with the application of psychiatric theories and practices for the care of patients with organic disease treated in hospitals and health care services (e.g., primary care). The request for assistance from colleagues belonging to other specializations is so high that since the mid 70's specific consultation-liaison units have been provided within the departments of psychiatry even more frequently.

Specific areas of Consultation Psychiatry include the study, the diagnosis, the treatment and the prevention of psychiatric morbidity in patients affected by organic disease or somatic symptoms.

The continuous attention to the phenomena of the organic and psychological interaction between body and mind is the operative height of the psychiatrist who operates outside the psychiatric setting [52]. The Consultation and Liaison Psychiatry has been defined as a branch of psychiatry that focuses on diagnosis, treatment, study and prevention of the psychiatric morbidity in patients with organic diseases and in those who have somatic symptoms. It also provides to organize psychiatric consultations, liaison activities and training for non-psychiatric operators who belong to all types of clinical setting, especially in the general hospital [53].

It must be said that the setting of the psychiatrist's intervention is very different from the usual one, and actually, it consists of a general hospital, inside of which should always be present a consultation-liaison unit and, inside of it, it must be present an ambulatory for the management of patients with chronic $C$ hepatitis and IFN treatment or at least, specialized and trained personnel to the management of this kind of patients.

In everyday clinical practice, psychiatric work consists of two types of intervention: the psychiatric consultation and liaison. The consultation focuses primarily on diagnosis and therapy. The diagnosis relies on means such as the clinical interview and psycho-diagnostic tests.

The treatment involves both pharmacologic and psychotherapy interventions.

The specificity of the consultant psychiatrist is therefore, based on his training that allows him to value the interactions between biological, psychological, social and psychodynamic variables in clinical practice, in diagnostics, in the treatment plan and prognosis of a mental disorder [49]. The consultant psychiatrist may also, if he deems it necessary, take charge of the patient in a continuous way, going beyond the single psychiatric consultation [54]. This possibility is not uncommon within the psychiatric ambulatories for the management of patients with HCV in IFN treatment. Many of these patients already have a basic psychiatric disorder, usually it is a medium degree disorder, never gone to the observation of psychiatrists. This psychiatric disorder will reoccur or worsen after the diagnosis of HCV or during IFN treatment, making continuous psychiatric cares necessary. In this regard, it should be said that patients are hopeful when it is given a chance of cure to them, on the other hand, they are frightened by the possibility of a therapeutic failure, the length of treatment and the possibility of serious side effects, including psychiatric symptoms. Actually, patients are really frightened of these latter symptoms because they have to face the spectrum of madness that, in their fantasies, drives them to the anguish of losing the ownership of their actions and thoughts. 
Fairly often when they come to the psychiatric interview they feel concerned, sometimes distrustful. They often hide their fears and try to be light-hearted and cheerful. The classic joke is: "Doctor, am I mad?

Whatever is the way they face the interview, the spectrum of madness, the anguish of losing the integrity of their mind, after having already lost their physical integrity, shine through their words. Sending patients to the psychiatry ambulatory is just a crucial moment. The hepatologist should always motivate the consultation request, properly informing the patient about the therapy that he is going to take. What is more, he should inform the patient of the possible psychiatric side effects, reassuring him at the same time. Reassuring a patient does not mean to say "do not worry, it's nothing" but it means to be empathetic and show understanding and affectivity to themselves. An empathetic attitude together with clarity of information and precision to show the therapeutic path, are interventions that reassure themselves the patient. It is important, therefore, to pursue an empathetic attitude, that is trying to get as close as possible to emotional experience and the experiences of the other person, trying to perceive feelings and personal meanings experienced by the patient and communicate him understanding. Empathic listening is, therefore, a psychological structure of hospitality that is used to establish trust and strong therapeutic alliance [55].

Clinical experience shows that all patients and, in particular the multi- diagnosed ones, get the most comfort from knowing that they are at the centre of an integrated and multidisciplinary system of interventions. Confirming them the importance of their centrality, building around the patient a set of integrated and coordinated interventions, certainly has positive effects on collaboration, motivation and, finally, on the outcomes of the interventions.

The Liaison is a more complex and continuous type of intervention, which arises from the request made by the doctors of department to the psychiatrist or to the clinical psychologist. It consists of a great collaboration with the treating team that requested the consultation in order to share the management of the patient's problems. The psychiatrist will discuss with colleagues from other specialties about therapeutic strategies, he will involve all health professionals in the care and in the project, he will deal with the relationship problems that may occur into the staff or between the team and the patient, he will work to promote biopsycho-social cultural models within the department. [56] Therefore, interventions addressed to one or more operators are the true liaison and they can focus on a specific case or on the overall method of intervention adopted by the consultants. Interventions vary from simple advice on the attitude to get in the therapeutic relationship to the interpretations of dynamics that may develop between the patient and the treating team, up to the training programs [57].

Motivations that drive the hepatologist to ask a psychiatric consultation are:

1. a consultation focused on the patient who has already started a therapy with interferon $\alpha$.

2. a consultation focused on the patient who is "candidate" for a therapy with interferon $\alpha$.

In the first case, the aim is the one of defining the clinical manifestations and, if possible, the formulation of the diagnosis and the treatment plan. 
In the second case, they are problematic patients since they have a personal story of psychiatric disorders, a story of past or current psychopharmacological treatments, a story of abuse or substance dependence. The consultation, therefore, has the purpose to set the patient from a diagnostic and personological point of view, in order to support the requesting colleague in his choice or orientating him towards different treatment choices. The psychiatrist could, for example, suggest to postpone, if possible, the starting of interferon therapy after an appropriate period of psychopharmacological treatment or even suggest to avoid interferon therapy. Any decision must be shared by the patient and, if possible, one member of his family at least. This kind of patients often needs to be taken in charge by the psychiatric service, for both pharmacological and psychotherapeutic aspects.

It is clear that each component of the medical team will manage the situation with important ethical implications. The ease of relationship, in terms of respect and recognition of roles, responsibilities and tasks within the team, is the secret for all health care professionals to take any decision with greater confidence.

Psychiatric consultation, therefore, can also be directed to the consultant: the patient will not be visited since the focus is a diagnostic, therapeutic and relational problem.

\section{Diagnosis and treatment of mental disorders in patients with $\mathrm{HCV}$ infection during interferon treatment}

In the following section we analyse the psychiatric symptoms most frequently found in these patients.

According to literature, many studies concerning the onset of psychiatric disorders in patients suffering from $\mathrm{HCV}$, show that depressive symptoms are commonly found despite of the assumption of antiviral therapy based on Interferon alpha (IFN $\alpha$ ) and Ribavirin [58]. It has been demonstrated that 35\% of 293 patients suffering from HCV reported an increase of the scores of depression even before starting antiviral therapy [59]. Also, it is well known the interferon $\alpha$ therapy may induce depressive symptoms.

The most important symptoms include: depressive symptoms, anxiety symptoms, cognitive symptoms, autonomic and somatic symptoms [11].

The autonomic and somatic symptoms tend to occur early during treatment with IFN- $\alpha$ and persist over time; the symptoms relating to mood, anxiety and cognitive symptoms tend to occur later during treatment $[60,61]$.

The onset of neuro-vegetative symptoms (e.g. psychomotor retardation) and depressive symptoms prior to antiviral treatment would increase the likelihood of developing depressive symptoms [62,63-64,65].

The management of patients with $\mathrm{HCV}$, candidate for therapy with interferon- $\alpha$, is a vexed question, considering the prevalence of hepatitis $C$, the likely presence of problems related to 
substance abuse, the psychological question that the impact of the diagnosis of a disease with poor prognosis determines and multi-systemic side effects that the therapy itself may cause.

Many studies have shown, in fact, that the impairment of quality of life in these patients is both linked to the severity of the disease [45] and to the previous substance abuse and side effects of treatment [66.67 ]. On the other hand, it is also true that interferon therapy, slowing down and, in some cases, arresting the progression of the disease, could improve the quality of life of patients suffering from hepatitis $C$ virus [68].

Psychiatric disorders more frequently found in $\mathrm{HCV}$ patients treated with interferon $\alpha$ include: sleep disorders, fatigue, irritability, anxiety disorders, cognitive disorders with impairment of concentration and memory, depressive episodes (mild, moderate or severe), confusion, delirium, psychotic disorder, mania, craving (alcohol, drugs). It is worth stressing that Depression does not just mean deflection of mood; this clinical picture, in fact, is characterized by a collection of disorders, involving emotional, cognitive and neuro-vegetative changes, which may occur in several combinations but usually tend to occur all together, resulting in the so-called depressive spectrum. Some studies have highlighted that depression related to interferon therapy is significantly different from Major Depression itself. The differences would involve the ideational sphere mainly. Patients on interferon therapy, in fact, do not present depressive ideations of guilt and ruin, ideas of inadequacy and worthlessness [69], typically experienced by patients with major depression. The hypothesis might be that depression occurring during interferon therapy should be included in the diagnostic category of "Mood Disorder due to a general medical condition" or "substance-induced disorder", according to the classification in the DSM IV TR [70 ]. According to the DSM IV TR, in fact, a Mood Disorder due to a general medical condition, is "a significant and persistent alteration in mood that is believed to be due to direct physiological effects of a general medical condition", including degenerative neurological diseases, metabolic conditions, viral infections or other infections as well. With regards to "Substances-Induced Mood Disorder", this is a condition that can be induced not only by drugs of abuse but also by medication, toxins. These conditions are not only characterized by a purely depressive symptomatology but also include symptoms such as high, expansive or irritable mood. It is shown that manic symptoms [71] and psychotic disorders [72] may also occur in patients receiving interferon.

It is also interesting to note that cognitive deficits often occur in patients suffering from chronic hepatitis $C[73,74]$.

It should also be noted that drug addicts with hepatitis $C$ are affected by psychopathological alterations to a far greater extent than those who did not abuse substances [75].

In the guidelines of the EASL (European Association for the Study of Liver) [76] it states that depression is the leading cause of discontinuation of antiviral treatment with interferon $\alpha$. According to these guidelines, patients with a documented story of depression should be evaluated by a psychiatrist before the start of antiviral therapy, so that it could be assessed the presence of possible psychiatric contraindications. These patients should be followed throughout the treatment to find out the onset of depression immediately and then, must be treated with antidepressants-based pharmacotherapy, when depressive symptoms are recognized. 
Some studies have evaluated the possible use of antidepressants, in particular SSRIs, for "preventative" purposes, showing actually a reduction in the incidence of depression during treatment with interferon- $\alpha$ [77].

Regarding the abuse of alcohol, the EASL guidelines recommend not to exclude alcoholic patients from any antiviral therapy, these patients should receive counselling interventions to reduce and then stop the consumption of alcohol, as well as psychological support in order to encourage the compliance with therapies. With regard to addicted patients, guidelines say that these patients should have stopped the assumption of drugs or should be taking replacement therapy from 6-12 months. In both cases, it is recommended a close follow up by a multidisciplinary team composed of the hepatologist, the psychiatrist and the psychologist.

The psychiatrist's figure in a multidisciplinary team for the care of patients with HCV is therefore essential to evaluate before beginning the treatment: the presence of current anxiety or depressive episode, history of anxiety or depression or other past psychiatric disorders, previous psychiatric hospitalisations, history of abuse or dependence on alcohol, drugs and medication, family history of anxiety disorders, depression or suicide attempts, the presence or absence of a social support network. Patient must be informed on the risks of psychiatric symptoms linked to the assumption of IFN- $\alpha$, showing him all treatment options that are available in case of psychiatric disorders. If psychiatric disorders were found under way, they should be treated before starting any antiviral therapy.

Self-administered tests can be used as diagnostic tools for psychiatric assessment, for example, the Zung Self Rating Depression Scale (ZSDS), the Zung Self Rating Anxiety Scale (ZSAS), other tests that can be administered are the Hamilton Depression Rating Scale (HAM D) and the Hamilton Anxiety Rating Scale (HAM A). These tests may be helpful to monitor patient during therapy.

Regarding any interventions to take in case of psychopathological disorders, such as anxiety disorders or depressive disorders, it should be said that they must be implemented as early as possible. The best first choice in treating anxiety disorders is the one of anxiolytics (BDZ). Among the benzodiazepine, those with little or no hepatic metabolism (oxazepam, lorazepam) should be chosen. A brief psychotherapeutic intervention may be decisive in case of reactive anxiety symptoms. SSRIs are the first choice for depressive disorders, especially paroxetine, sertraline, citalopram, that is, those with the best tolerability profile, the fewer interactions, the best demonstrated efficiency. If the severity of depressive condition is mild or moderate, antiviral treatment can be continued. Even in this case psychotherapeutic support interventions are suggested. If depressive symptoms persist and therapy does not work (by 2-4 weeks), it should be considered the use of another antidepressant with a different mechanism of action, or the reduction or discontinuation of IFN treatment; this decision could be made jointly by the infectious disease specialist, the consultant psychiatrist, the patient and a familiar at least. If psychotic symptoms occurred, IFN therapy should be discontinued and, if necessary, started again, only after the patient has been properly treated with antipsychotic drugs.

Depression induced by IFN $\alpha$ responds well to treatment with SSRIs, as it would be mainly due to alterations involving the serotonergic circuits, while the so-called Neuro-vegetative 
Syndrome induced by IFN, characterized by fatigue, anorexia, pain and psychomotor retardation, which is often found in association with depressive symptoms, would benefit from drugs such as SNRIs (venlafaxine) and Bupropion [78].

Confusional episodes characterized by disorientation, somnolence, psychomotor retardation, psychotic symptoms have also been documented in patients treated with IFN $\alpha[79,80]$. In these cases it is necessary to discontinue therapy and administer neuroleptics or atypical antipsychotics, including especially olanzapine[81].

It should also be considered the degree of hepatic impairment in these patients in order to determine the optimal dosage of the drugs. In general, drugs should be used at the lowest effective dose. It should be pointed out that paroxetine, fluoxetine and fluvoxamine may reduce the metabolism of other drugs, increasing their blood levels.

It is also useful to consider that some side effects of antidepressants may be exploited for the patient's benefit; such as weight gain and drowsiness that occurred during treatment with mirtazapine may be useful in those patients affected by HCV, in which therapy with IFN $\alpha$ and ribavirin caused hyporexia / anorexia and insomnia.

Another psychiatric symptom that may be found during therapy with IFN $\alpha$, as already said, is mania $[82,83]$. It is important to note that irritability which very often occurs in these patients is a classic symptom of mania [84]. It seems that manic or hypomanic symptoms develop in approx. $20 \%$ of HCV-positive patients treated with IFN- $\alpha$ and ribavirin [85]. It is very important to recognize dysphoric mania carefully. It is a clinical situation in which both elements related to manic activation and depressive elements may occur together. The use of antidepressants in dysphoric mania would induce the worsening of the manic picture. Indeed, it is common that dysphoria may be sometimes confused with a depressive symptom and that is why the opinion of the psychiatrist should be asked whenever a picture of psychopathological alterations is suspected.

Finally, we must not forget that interferon therapy may increase the risk of suicide although studies in this field are still few and results don't appear unique [86, 87]. It is clear that the prognosis of viral disease itself, which, as well known, can result in cirrhosis and hepatocellular carcinoma, may cause psychological reactions of despair that can lead to depression and suicidal ideation. It is worth noting that, according to the DSM IV TR "Mood Disorder due to a general medical condition increases the risk of suicide attempts and suicide" [1]. Even in case of suicide then, we must consider several risk factors, ranging from psychological consequences of diagnosis and prognosis to depression caused by the therapy.

\section{Conclusions}

In view of these considerations, it is understandable that the management of patients with $\mathrm{HCV}$ treated or not with IFN $\alpha$, requires several specialized figures that can deal with the many aspects that this condition brings with it. The guidelines and literature recommend the 
presence of a multidisciplinary team, in which the psychiatrist plays a key role for the great incidence of psychiatric disorders in these patients.

In a Consensus Conference held by European specialists in 2005 it was established that, in case of psychiatric disorders, it is not necessary to delay or forego treatment if this is complemented by a global care, where several specialists are involved, such as: Gastroenterologist, Infectious disease specialist, Psychiatrist, Addiction Service Operators, Psychologist - Counsellor [88]. Then, in case of psychiatric disorders, treatment with IFN is strongly recommended, keeping in mind that the psychological state must be stabilized before starting antiviral therapy.

Some studies have shown that psychiatric disorders may also occur several months after discontinuation of therapy with IFN $\alpha$ and ribavirin [89], or may still persist even after discontinuation of therapy [90]. It would be good practice then, that the control of the mental state of patient continued even after discontinuation of antiviral treatment.

In view of these considerations, it is certain that interferon therapy acts causing mental status changes that include mainly changes in mood and anxiety. The widespread of $C$ virus is a ubiquitous problem and therefore, it requires a strong attention that helps to do more and more to deal with any organic and psychic problems "orbiting" around this condition. It would be desirable that these patients may have assistance which allows them to continue antiviral therapy, coping with possible side effects that would probably be a contraindication to continue therapy, "condemning" the patient to a forced coexistence with the virus.

\section{Author details}

Giuseppe Valerio Torres ${ }^{1}$, Emanuela Apicella², Elisa D'Alterio ${ }^{3}$, Angela Chiara Cecere ${ }^{2}$ and Antonello Bellomo ${ }^{4}$

1 Department of Mental Health ASL FG, University of Foggia, Italy

2 University of Foggia, Italy

3 University of Naples, Italy

4 Department of Medical Occupational Sciences, University of Foggia, Italy

\section{References}

[1] Diagnostic, A. A. V. V. and statistical manual of mental disorder: DSM IV TR. American Psychiatric Pub. $4^{\mathrm{a}}$ ed;(2000).

[2] Lteif, G. N, \& Mavissakalion, M. R. Life events and panic disorders/agoraphobia. Compreh. Psychiatry (1995). 
[3] Kessler, R. C. Sociology and Psychiatry. In: Kaplan H.I., Sadock B.J. Comprehensive Textbook of Psychiatry. Williams \& Wilkins; (1989).

[4] Paykel, E. S. The interview for recent life events. Psycol. Med (1997). , 27(2), 301-310.

[5] Carta, M. G. Hardoy MC at al. Association of chronic hepatitis $C$ with major depressive disorders: irrespective of interferon-alpha therapy. Clinical Practice and Epidemiology in Mental Health (2007).

[6] Voss, J. G. Dodd, Portillo C, Holzemer W. Theories of fatigue: application in HIV/AIDS. J. Assoc. Nurses AIDS Care (2006). , 17-37.

[7] Astle, F. Diabetes and depression: a review of the literature. Nurs Clin North Am. (2007). , 42-67.

[8] Gold, S. M, \& Irwin, M. R. Depression and immunity: inflammation and depressive symptoms in multiple sclerosis. Neurol. Clin.(2006). , 24-507.

[9] Ranjit, N, Diez-roux, A. V, Shea, S, Cushman, M, Seeman, T, Jackson, S. A, \& Ni, H. Psychosocial factors and inflammation in the multi-ethnic study of atherosclerosis. Arch. Intern. Med. (2007). , 167-174.

[10] Dieperink, E, Willenbring, M, \& Ho, S. B. Neuropsychiatric symptoms associated with hepatitis C and interferon alpha: a review. Am J Psychiatry (2000). , 157-867.

[11] Lotrich, F. E, Rabinovitz, M, et al. Depression following pegylated interferon-alpha: Characteristics and vulnerability. Journal of Psychosomatic Research (2007). , 63-131.

[12] Licinio, J, \& Wong, M. L. The role of inflammatory mediators in the biology of major depression: central nervous system cytokines modulate the biological substrate of depressive symptoms, regulate stress-responsive systems, and contribute to neurotoxicity and neuroprotection. Mol. Psychiatry (1993). , 4-317.

[13] Loftis, J. M, \& Hauser, P. The phenomenology and treatment of interferoninduced depression. J. Affect Disord. (2004). , 82-175.

[14] Raison, C. L, Capuron, L, \& Miller, A. H. Cytokines sing the blues: inflammation and the pathogenesis of depression. Trends Immunol. (2006). , 27-24.

[15] Schiepers, O. J, Wichers, M. C, \& Maes, M. Cytokines and major depression. Prog. Neuropsychopharmacol. Biol. Psychiatry (2005). , 29-201.

[16] Andreoli, A. V, Keller, S. E, Rabaeus, M, Marin, P, Bartlett, J. A, \& Taban, C. Depression and immunity: age, severity, and clinical course. Brain. Behav. Immun. (1993). , 7-279.

[17] Leonard, B. E, \& Myint, A. The psychoneuroimmunology of depression. Hum Psychopharmacol (2009). Apr;, 24(3), 165-175.

[18] Turnbull, A. V, \& Rivier, C. L. Regulation of the hypothalamic-pituitary-adrenal axis by cytokines: actions and mechanisms of action. Physiol Rev (1999). Jan;, 79(1), 1-71. 
[19] Friebe, A, Schwarz, M. J, Schmid-wendtner, M, Volkenandt, M, Schmidt, F, Horn, M, Janssen, G, \& Schaefer, M. Pre-treatment levels of sTNF-R1 and sIL-6R are associated with a higher vulnerability for IFN-alpha-induced depressive symptoms in patients with malignant melanoma. J. Immunother (1997). , 30-333.

[20] Musselman, D. L, Miller, A. H, Porter, M. R, Manatunga, A. K, Gao, F, Penna, S, Pearce, B. D, Landry, J, Glover, S, Mcdaniel, J. S, \& Nemeroff, C. B. Higher than normal plasma interleukin-6 concentrations in cancer patients with depression: preliminary findings. Am. J. Psychiatry (2001). , 158-1252.

[21] Wichers, M. C, Kenis, G, Leue, C, Koek, G, Robaeys, G, \& Maes, M. Baseline immune activation as a risk factor for the onset of depression during interferon-alpha treatment. Biol. Psychiatry (2006). , 60-77.

[22] Morasco, B. J, \& Rifai, M. A. A randomized trial of paroxetine to prevent interferon- $\alpha-$ induced depression in patients with hepatitis C. Journal of Affective Disorders (2007). , 103-83.

[23] Musselman, D. L, Lawson, D. H, Gumnick, J. F, Manatunga, A. K, Penna, S, Goodkin, R. S, Greiner, K, Nemeroff, C. B, \& Miller, A. H. Paroxetine for the prevention of depression induced by high-dose interferon alpha. N. Engl. J. Med. (2001). , 344, 961-966.

[24] Dantzer, R, Connor, O, Freund, J. C, Johnson, G. G, \& Kelley, R. W. K.W. From inflammation to sickness and depression: when the immune system subjugates the brain. Nat. Rev. Neurosci. (2008). , 9-46.

[25] Capuron, L, Ravaud, A, Neveu, P. J, Miller, A. H, Maes, M, \& Dantzer, R. Association between decreased serum tryptophan concentrations and depressive symptoms in cancer patients undergoing cytokine therapy. Mol. Psychiatry (2002b). , 7-468.

[26] Maes, M, Bonaccorso, S, Marino, V, Puzella, A, Pasquini, M, Biondi, M, Artini, M, Almerighi, C, \& Meltzer, H. Treatment with interferon-alpha (IFN alpha) of hepatitis C patients induces lower serum dipeptidyl peptidase IV activity, which is related to IFN alpha-induced depressive and anxiety symptoms and immune activation. Mol. Psychiatry (2001). , 6-475.

[27] Miller, A. H. Mechanisms of cytokine-induced behavioral changes: Psychoneuroimmunology at the translational interface. Brain, Behavior, and Immunity (2009). , 23-149.

[28] Zhu, C. B, Carneiro, A. M, Dostmann, W. R, Hewlett, W. A, \& Blakely, R. D. p. MAPK activation elevates serotonin transport activity via a traffickingindependent, protein phosphatase 2A-dependent process. J. Biol. Chem. (2005). , 280-15649.

[29] Zhu, C. B, Blakely, R. D, \& Hewlett, W. A. The proinflammatory cytokines interleukin-1beta and tumor necrosis factor-alpha activate serotonin transporters. Neuropsychopharmacology (2006). , 31-2121.

[30] Willeit, M, Sitte, H. H, Thierry, N, Michalek, K, Praschak-rieder, N, Zill, P, Winkler, D, Brannath, W, Fischer, M. B, Bondy, B, Kasper, S, \& Singer, E. A. Enhanced serotonin 
transporter function during depression in seasonal affective disorder. Neuropsychopharmacology (2008). , 33, 1503-1513.

[31] Majer, M, Welberg, L. A, Capuron, L, Pagnoni, G, Raison, C. L, \& Miller, A. H. IFNalphainduced motor slowing is associated with increased depression and fatigue in patients with chronic hepatitis C. Brain Behav. Immun. (2008). , 22-870.

[32] Horikawa, N, Yamazaki, T, Sagawa, M, \& Nagata, T. A case of akathisia during interferon-alpha therapy for chronic hepatitis type C. Gen. Hosp. Psychiatry (1999). , 21-134.

[33] Kamata, M, Higuchi, H, Yoshimoto, M, Yoshida, K, \& Shimizu, T. Effect of single intracerebroventricular injection of alpha-interferon on monoamine concentrations in the rat brain. Eur. Neuropsychopharmacol. (2000). , 10-129.

[34] Salamone, J. D, Correa, M, Mingote, S. M, \& Weber, S. M. Beyond the reward hypothesis: alternative functions of nucleus accumbens dopamine. Curr. Opin. Pharmacol. (2005). , 5-34.

[35] Schultz, W. Multiple dopamine functions at different time courses. Annu. Rev. Neurosci. (2007). , 30-259.

[36] Kitagami, T, Yamada, K, Miura, H, Hashimoto, R, Nabeshima, T, \& Ohta, T. Mechanism of systemically injected interferon-alpha impeding monoamine biosynthesis in rats: role of nitric oxide as a signal crossing the blood-brain barrier. Brain Res. (2003). , 978-104.

[37] Capuron, L, Neurauter, G, Musselman, D. L, Lawson, D. H, Nemeroff, C. B, Fuchs, D, \& Miller, A. H. Interferon-alpha-induced changes in tryptophan metabolism. Relationship to depression and paroxetine treatment. Biol. Psychiatry (2003a). , 54-906.

[38] Wu, H. Q, Rassoulpour, A, \& Schwarcz, R. Kynurenic acid leads, dopamine follows: a new case of volume transmission in the brain? J. Neural. Transm. (2007). , 114, 33-41.

[39] Moron, J. A, Zakharova, I, Ferrer, J. V, Merrill, G. A, Hope, B, Lafer, E. M, Lin, Z. C, Wang, J. B, Javitch, J. A, Galli, A, \& Shippenberg, T. S. Mitogen-activated protein kinase regulates dopamine transporter surface expression and dopamine transport capacity. J. Neurosci. (2003). , 23, 8480-8488.

[40] Capuron, L, Pagnoni, G, Demetrashvili, M, Woolwine, B. J, Nemeroff, C. B, Berns, G. $\mathrm{S}, \&$ Miller, A. H. Anterior cingulate activation and error processing during interferonalpha treatment. Biol. Psychiatry (2005). , 58, 190-196.

[41] Reichenberg, A, Yirmiya, R, Schuld, A, Kraus, T, Haack, M, Morag, A, \& Pollmacher, T. Cytokine-associated emotional and cognitive disturbances in humans. Arch. Gen. Psychiatry (2001). , 58, 445-452. 
[42] Ursu, S, Stenger, V. A, Shear, M. K, Jones, M. R, \& Carter, C. S. Overactive action monitoring in obsessive-compulsive disorder: evidence from functional magnetic resonance imaging. Psychol. Sci. (2003). , 14, 347-353.

[43] Grassi, L, Satriano, J, Serra, A, Biancosino, B, Zotos, S, Sighinolfi, L, \& Ghinelli, F. Emotional stress, psychosocial variables and coping associated with haepatitis $C$ virus and human immunodeficiency virus infections in intravenous drug users. Psychother Psychosom (2002). , 71-342.

[44] Carta, M. G. Hardoy MC at al. Association of chronic hepatitis C with major depressive disorders: irrespective of interferon-alpha therapy. Clinical Practice and Epidemiology in Mental Health (2007).

[45] Forton, D. M, Allsop, J. M, Main, J, Foster, G. R, Thomas, H. C, \& Taylor-robinson, S. D. Evidence for a cerebral effect of the hepatitis C virus. Lancet (2001). , 358-38.

[46] Chang, L, Ernst, T, Witt, M. D, Ames, N, Gaiefsky, M, \& Miller, E. Relationships among brain metabolites, cognitive function, and viral loads in antiretroviral-naive HIV patients. Neuroimage (2002). , 17-1638.

[47] Chang, L, Lee, P. L, Yiannoutsos, C. T, Ernst, T, Marra, C. M, Richards, T, Kolson, D, Schifitto, G, Jarvik, J. G, Miller, E. N, Lenkinski, R, Gonzalez, G, \& Navia, B. A. HIV MRS Consortium: A multicenter in vivo proton- MRS study of HIV-associated dementia and its relationship to age. Neuroimage (2004). , 23-1336.

[48] Thomas, H. C, Torok, M. E, Forton, D. M, \& Taylor-robinson, S. D. Possible mechanisms of action and reasons for failure of antiviral therapy in chronic hepatitis C. J Hepatol (1999). , 31(1), 152-159.

[49] Radkowski, M, Wilkinson, J, Nowicki, M, Adair, D, Vargas, H, Ingui, C, Rakela, J, \& Laskus, T. Search for hepatitis $C$ virus negative-strand RNA sequences and analysis of viral sequences in the central nervous system: evidence of replication. J Virol (2002). , 76-600.

[50] Shepherd, J, Brodin, H, Cave, C, Waugh, N, Price, A, \& Gabbay, J. Pegylated interferon-2a and-2b in combination with ribavirin in the treatment of chronic hepatitis $C: a$ systematic review and economic evaluation. Health Technology Assessment (2004).

[51] Invernizzi, G, Gala, C, \& Bressi, C. La Psichiatria di Consultazione nell'Ospedale Generale. CIC Ed. Internazionali, Roma;(1997).

[52] De Bertolini, C, Rigatelli, M, \& Rizzardo, R. Psichiatria di consultazione e collegamento. In:Pavan L. Trattato italiano di psichiatria. Masson;(1999).

[53] Lipowski, Z. J. Consultation-Liaison Psychiatry at century's end. Psychosomatics (1992).

[54] Kaplan, H. I, Sadoch, B. J, \& Grebb, J. A. Psichiatria.Manuale di scienze del comportamento e psichiatria clinica. Centro Scientifico Internazionale (2001). , 818-819. 
[55] Orsi, E. Comunicazione efficace: l'approccio empatico nelle relazioni d'aiuto. In: Bellomo A. Psicologia Medica e abilità relazionali. Edizioni Minerva Medica (2004). , 79-80.

[56] Mayou, R. A. Consultation-liaison psychiatry: panoramica internazionale. Upiohn International inc (1988).

[57] Fulcheri, M, Bellino, S, \& Zizza, M. Di Lorenzo R, Bogetto F. La Psichiatria di consultazione e collegamento::modelli e dati della letteratura. Rivista di Psichiatria (2001).

[58] Angelino, A. F, \& Treisman, G. J. Evidence-informed assessment and treatment of depression in HCV and interferon-treated patients. Int. Rev. Psychiatry (2005). , 17-471.

[59] Fireman, M, Indest, D. W, Blackwell, A, Whitehead, A, \& Hauser, P. Addressing trimorbidity (hepatitis C, psychiatric disorders, and substance use): the importance of routine mental health screening as a component of a comanagement model of care. Clin. Infect. Dis. (2005). , 40(5), 286-291.

[60] Capuron, L, Gumnick, J. F, Musselman, D. L, Lawson, D. H, Reemsnyder, A, Nemeroff, C. B, \& Miller, A. H. Neurobehavioral effects of interferon-alpha in cancer patients: phenomenology and paroxetine responsiveness of symptom dimensions. Neuropsychopharmacology (2002). , 26-643.

[61] Capuron, L, \& Miller, A. H. Cytokines and psychopatology :lessons from interferonalpha.Biol.Psichiatry (2004). , 56-819.

[62] Capuron, L, \& Miller, A. H. Cytokines and psychopathology: lessons from interferonalpha. Biol. Psychiatry (2004). Capuron L, Ravaud A, Dantzer R. Timing and specificity of the cognitive changes induced by interleukin-2 and interferon-alpha treatments in cancer patients. Psychosom. Med. 2001;63 376-386., 56-819.

[63] Capuron, L, \& Ravaud, A. Prediction of the depressive effects of interferon alpha therapy by the patient's initial affective state. N. Engl. J. Med. (1999).

[64] Majer, M, Welberg, L. A, Capuron, L, Pagnoni, G, Raison, C. L, \& Miller, A. H. IFNalphainduced motor slowing is associated with increased depression and fatigue in patients with chronic hepatitis C. Brain Behav. Immun. (2008). , 22-870.

[65] Raison, C. L, Borisov, A. S, Broadwell, S. D, Capuron, L, Woolwine, B. J, Jacobson, I. M, Nemeroff, C. B, \& Miller, A. H. Depression during pegylated interferonalpha plus ribavirin therapy: prevalence and prediction. J. Clin. Psychiatry (2005). , 66-41.

[66] Hussain, K, Fontana, R, Moyer, C, Su, G. L, Sneed-pee, N, \& Lok, A. S. F. Comorbid illness is an important determinant of health-related quality of life in patients with chronic hepatitis C. Am J Gastroenterol. (2001). , 96-2737.

[67] Spiegel, B, Younossi, Z, Hays, R, Hays, R. D, Revicki, D, Robins, S, et al. Impact of hepatitis $C$ on health related quality of life: a systematic review and quantitative assessment. Hepatology. (2005). , 41-790. 
[68] Kamal, S. M, Ahmed, A, et al. Enhanced efficacy of pegylated interferon alpha-2a over pegylated interferon and ribavirin in chronic hepatitis $C$ genotype $4 \mathrm{~A}$ randomized trial and quality of life analysis. Liver Int. (2011). Mar;, 31(3), 401-11.

[69] Pasquini, M, Speca, A, et al. Differences in depressive thougths between major depressive disorder, IFN- $\alpha$-induced depression, and depressive disorders among cancer patients. Journal of Psychosomatic Research (2008). , 65-153.

[70] Gregory, M, et al. Interferon-Induced Depression in CHC. J Clin Gastroenterol. (2006).

[71] Giunta, B, Somboonwit, C, et al. Psychiatric implications of hepatitis-C infection. Crit Rev Neurobiol. (2007).

[72] Silverman, B. C, Kim, A. Y, \& Freudenreich, O. Interferon-induced psychosis as a "psychiatric contraindication" to hepatitis $C$ treatment: a review and case-based discussion. Psychosomatics. (2010). Jan-Feb; 51(1), 1-7.

[73] Forton, D. M, Thomas, H. C, et al. Hepatitis C and cognitive impairment in a cohort of patients with mild liver disease. Hepatology (2002). , 3-433.

[74] Forton, D. M, Taylor-robinson, S. D, \& Thomas, H. C. Cerebral dysfumction in chronic hepatitis C infection. J Viral Hepatitis (2003). , 10-81.

[75] Johnson, M. E, Fisher, D. G, et al. Hepatitis C virus and depression in drug users. Am J Gastroenterol. (1998). , 93-785.

[76] European Association for the Study of the LiverEASL Clinical Practice Guidelines: Management of hepatitis C virus infection. Journal of Hepatology (2011).

[77] Bezemer, G, Van Gool, A. R, Drenth, J. P, \& Hansen, B. E. Fortuyn HAD, Weegink CJ, et al. A double blind, placebo-controlled trial with escitalopram to prevent psychiatric adverse events during treatment with pegylated interferonalpha and ribavirin for chronic hepatitis c: the "prevention of psychiatric side effects (Pops)-study". Hepatology (2008). A.

[78] Charles, L. Raison Marina Demetrashvili, Lucile Capuron and Andrew H. Miller. Neuropsychiatric Adverse Effects of Interferon- $\alpha$ Recognition and Management. CNS Drugs. (2005). , 19(2), 105-123.

[79] Rohatiner, A. Z, Prior, P. F, Burton, A. C, et al. Central nervous system toxicity of interferon. Br J Cancer. (1983). , 47(3), 419-22.

[80] Poutiainen, E, Hokkanen, L, Niemi, M. L, et al. Reversible cognitive decline during high-dose $\alpha$-interferon treatment. Pharmacol Biochem Behav. (1994). , 47(4), 901-5.

[81] Hoffman, R. G, Cohen, M. A, Alfonso, C. A, et al. Treatment of interferon-induced psychosis in patients with comorbid hepatitis C and HIV. Psychosomatics. (2003). , 44(5), 417-20. 
[82] Carpiniello, B, Orru, M. G, Baita, A, et al. Mania induced by withdrawal of treatment with interferon alfa. Arch Gen Psychiatry. (1998). , 55(1), 88-9.

[83] Kanno, A, Yamada, M, Abe, M, et al. A case of interferon $\alpha$-induced manic psychosis in chronic hepatitis C. Tohoku J Exp Med. (1999). , 187(1), 79-82.

[84] Raison, C. L, \& Miller, A. H. The neuroimmunology of stress and depression. Semin Clin Neuropsychiatry (2001). , 6(4), 277-94.

[85] Gould, R. A, Ball, S, Kaspi, S. P, et al. Prevalence and correlates of anger attacks: a two site study. J Affect Disord. (1996). , 39(1), 31-8.

[86] Sockalingam, S, Links, P. S, \& Abbey, S. E. Suicide risk in hepatitis C and during interferon-alpha therapy: a review and clinical update. J Viral Hepat. (2011). Mar;, 18(3), 153-60.

[87] Debien, C. De Chouly De Lenclave MB, Foutrein P, Bailly D. [Alpha-interferon and mental disorders]. Encephale. (2001). Jul-Aug;, 27(4), 308-17.

[88] Alberti, A, Clumeck, N, Collins, S, Gerlich, W, Lundgren, J, Palù, G, Reiss, P, Thiebaut, R, Weiland, O, \& Yazdanpanah, Y. Zeuzem S (The ECC Jury). Short statement of the first European consensus conference on the treatment of chronic hepatitis B and $\mathrm{C}$ in HIV co-infected patients. Journal of Hepatology (2005). , 42-615.

[89] Nickel, T, et al. Depression during therapy with interferon alpha-how long should an antidepressant treatment last? Pharmacopsychiatry (2005). , 38-102.

[90] Meyers, C. A, et al. Persistent neurotoxicity of systemically administered interferonalpha. Neurology (1991). , 41-672. 